\title{
Differential expression of PTEN in hepatic tissue and hepatic stellate cells during rat liver fibrosis and its reversal
}

\author{
LIBO ZHENG $^{1 *}$, XIULI CHEN ${ }^{1,2^{*}}$, JINBO GUO $^{1}$, HUICONG SUN $^{1}$, \\ LEI LIU ${ }^{1}$, DAVID Q. SHIH ${ }^{3}$ and XIAOLAN ZHANG ${ }^{1}$ \\ ${ }^{1}$ Department of Gastroenterology, The Second Hospital of Hebei Medical University, Hebei Key Laboratory of \\ Gastroenterology, Hebei Institute of Gastroenterology; ${ }^{2}$ The Fifth Hospital of Shijiazhuang, Shijiazhuang, \\ Hebei 050000, P.R. China; ${ }^{3}$ Inflammatory Bowel and Immunobiology Research Institute, \\ Cedars-Sinai Medical Center, Los Angeles, CA, USA
}

Received July 15, 2012; Accepted September 7, 2012

DOI: 10.3892/ijmm.2012.1151

\begin{abstract}
To evaluate the change in phosphatase and tensin homology deleted on chromosome ten (PTEN) expression in liver fibrogenesis, particularly the reversal of fibrogenic liver tissues, and to investigate the relation with the proliferation and apoptosis of hepatic stellate cells (HSCs) in vivo, a rat model of hepatic fibrosis was established by hypodermic injection of carbon tetrachloride $\left(\mathrm{CCl}_{4}\right)$ mixed with olive oil at the concentration of $40 \%$ for 5 weeks $(2 \mathrm{ml} / \mathrm{kg}$, twice a week). Reversal of fibrosis was achieved with normal feedings for 4 weeks after $\mathrm{CCl}_{4}$ injection for 5 weeks. The expression of PTEN was measured by immunofluorescence, western blot analysis and real-time PCR. Co-expression of $\alpha$-smooth muscle actin ( $\alpha$-SMA) with PTEN and $\alpha$-SMA with terminal deoxynucleotidyltransferase-mediated dUTP nick end labelling (TUNEL) were assessed by confocal laser scanning microscopy. The results displayed that the expression of PTEN was reduced with fibrosis in both rat liver tissues and activated HSCs. By contrast, PTEN expression was increased with the reversal of liver fibrosis. Compared to the fibrogenic state, there were increased numbers of apoptotic activated HSCs during reversal of fibrosis. These data suggest that the dynamic expression of PTEN in rat liver tissues is negatively correlated with liver fibrosis and activated HSCs and is positively correlated with reversal of fibrosis and apoptotic
\end{abstract}

Correspondence to: Professor Xiaolan Zhang, Department of Gastroenterology, The Second Hospital of Hebei Medical University, Hebei Key Laboratory of Gastroenterology, Hebei Institute of Gastroenterology, No. 215 Heping West Road, Shijiazhuang, Hebei 050000, P.R. China

E-mail: xiaolanzh@126.com

*Contributed equally

Key words: hepatic fibrosis, reversal, phosphatase and tensin homology deleted on chromosome ten, hepatic stellate cells, proliferation, apoptosis activated HSCs. Modulation of PTEN expression may be an effective and novel method for the treatment of liver fibrosis.

\section{Introduction}

Chronic liver inflammation represents a major driving force for progressive extracellular matrix (ECM) accumulation, leading to liver cirrhosis with resultant complications that include portal hypertension and hepatocellular carcinoma $(1,2)$. The activation of hepatic stellate cells (HSCs) is one of the critical events involved in hepatic fibrosis (3). Previously, the reduction and/or reversal of liver fibrosis were shown to be associated with apoptosis of activated HSCs $(4,5)$.

Phosphatase and tensin homology deleted on chromosome ten (PTEN) is a tumor-suppressor gene with double phosphatase activities (6). Recent studies have demonstrated that the expression of PTEN is decreased in the myofibroblasts of lung tissues in idiopathic pulmonary interstitial fibrosis. PTEN has been shown in vitro to negatively regulate the differentiation of myofibroblasts (7). Additionally, we previously showed that the expression of PTEN is reduced in activated HSCs in fibrotic hepatic tissues in vivo (8). Conversely, the upregulation of PTEN may induce apoptosis in activated HSCs in vitro (9).

Carbon tetrachloride $\left(\mathrm{CCl}_{4}\right)$ is widely used to experimentally induce liver injury in rodents. Its prolonged administration leads to liver fibrosis, cirrhosis and hepatocellular carcinoma. Reversal of liver fibrosis occurs with $\mathrm{CCl}_{4}$ discontinuation (10). This model has been used extensively to examine the pathogenesis of cirrhosis.

In the current study, we exposed rats to $\mathrm{CCl}_{4}$ and discovered that PTEN expression was reduced in activated HSCs during liver fibrogenesis. Upon withdrawal of $\mathrm{CCl}_{4}, \mathrm{PTEN}$ expression was increased in apopotic activated HSCs with the reversal of liver fibrosis. Our results suggest that PTEN may be an important therapeutic target for the management of liver fibrosis.

\section{Materials and methods}

Animal models. One hundred and eight adult male Wistar rats weighing 350-450 g were obtained from the Experimental Animal Center of Hebei Medical University. The research was 
conducted in accordance with the internationally accepted principles for laboratory animal use and care as found in the US guidelines (NIH publication \#85-23, revised in 1985). The experiment was performed in compliance with the national ethical guidelines for the care and use of laboratory animals (certificate no. 911102).

A rat model of hepatic fibrosis was established by hypodermic injection of $\mathrm{CCl}_{4}$ mixed with olive oil at the concentration of $40 \%$ (Huarui Scientific \& Technological Co., Shijiazhuang, China) for 5 weeks ( $2 \mathrm{ml} / \mathrm{kg}$, twice a week); the reversal model was established through 4 weeks of normal feedings based on hypodermic injection of $40 \% \mathrm{CCl}_{4}$ for 5 weeks $(2 \mathrm{ml} / \mathrm{kg}$, twice a week). One hundred and eight male Wistar rats were randomly divided into the following groups ( $\mathrm{n}=6$ in each group): model group (containing $1,2,3$, 4 and 5 week groups), model control group, reversal group (containing Re 1, 2, 3 and 4 week groups), and the reversal control group. Rats were sacrificed at the indicated times and their livers were harvested for subsequent analysis.

Histopathology. Liver specimens were fixed $12-24 \mathrm{~h}$ in $4 \%$ phosphate-buffered paraformaldehyde (Huarui Scientific \& Technological Co.) and then embedded in paraffin for light microscopy examination. Tissue sections (4- $\mu$ m thick) were stained with hematoxylin and eosin (H\&E) for morphological evaluation and Masson trichrome (MT) for assessing the degree of fibrosis.

Immunofluorescent detection of PTEN and $\alpha$-SMA. Immunofluorescent studies were performed on $4-\mu \mathrm{m}$ frozen sections. Briefly, the sections were fixed by $4 \%$ phosphate-buffered paraformaldehyde and washed with $0.1 \%$ Triton X-100 TBS (TBSTx). Five percent bovine serum albumin (BSA) TBSTx was used as a sealed liquid and then the specimens were incubated overnight at $4^{\circ} \mathrm{C}$ with the primary antibody (either mouse anti-PTEN monoclonal antibody at a dilution of 1:100 or rabbit anti- $\alpha$-SMA monoclonal antibody at a dilution of 1:200) (Santa Cruz Biotechnology, Inc., Santa Cruz, CA, USA). After the sections were washed, the Cy3-labeled goat anti-mouse IgG secondary antibody or FITC-labeled goat anti-rabbit (1:400 dilution) (Beyotime Institute of Biotechnology, Shanghai, China) was added and the sections were incubated at $37^{\circ} \mathrm{C}$ for $1 \mathrm{~h}$. DAPI was used for nuclear staining. The negative control samples were processed under the same conditions, except that 5\% BSA TBSTx was used in place of the primary antibody. The $\alpha$-SMA and PTEN-positive expression levels were measured by a Motic Med 6.0 digital video image analysis system (Motic China Group Co., Ltd., Xiamen, China) and expressed as optical density values.

Immunofluorescence double labeling confocal laser scanning microscopy of PTEN and $\alpha$-SMA. The primary antibodies used were mouse anti-PTEN monoclonal (1:100) and rabbit anti- $\alpha$-SMA monoclonal (1:200) antibodies, and the secondary antibodies were avidin-Cy3-labeled goat anti-mouse and FITC-labeled goat anti-rabbit antibodies. Sections underwent the same washing, blocking and primary detection procedure as mentioned above. The co-expression of PTEN and $\alpha$-SMA was observed using a confocal laser scanning microscope.
PTEN-positive expression appeared as red fluorescent foci, $\alpha$-SMA-positive expression appeared as green fluorescent foci and the colocalization of the two markers appeared as yellow fluorescent foci.

Immunofluorescence double labeling confocal laser scanning microscopy of TUNEL and $\alpha$-SMA. Immunofluorescent studies were performed on $4-\mu \mathrm{m}$ frozen sections. The sections were fixed by $4 \%$ phosphate-buffered paraformaldehyde and washed with PBS. Five percent BSA TBSTx was used as a sealed liquid, and the specimens were then incubated overnight at $4^{\circ} \mathrm{C}$ with the primary antibody mouse anti- $\alpha$-SMA polyclonal (1:100 dilution). The Cy3-labeled goat anti-mouse IgG secondary antibody (1:400 dilution) and terminal deoxynucleotidyltransferase-mediated dUTP nick end labelling (TUNEL) liquid solution (TdT enzyme $2 \mu \mathrm{l}+$ fluorescent marker liquid $48 \mu \mathrm{l}$ for each section) (Beyotime Institute of Biotechnology) were added and the sections were incubated for $1 \mathrm{~h}$. DAPI was used for nuclear staining. The negative control samples were processed under the same conditions, except that 5\% BSA TBSTx was used in place of the primary antibody and TdT enzyme. The apoptotic cells appeared as green fluorescent foci by TUNEL kit and $\alpha$-SMA-positive expression appeared as red fluorescent foci and colocalization of the two markers appeared as yellow fluorescent foci. The apoptotic index was calculated as the ratio of yellow cells to total green cells.

Western blot analysis. Primary antibodies used were mouse anti-PTEN monoclonal (1:200) and rabbit anti-GAPDH polyclonal (1:500) antibodies. Western blotting was performed as previously described (8).

Real-time fluorescent quantitation PCR assay. The total RNA of hepatic tissue was extracted with the TRIzol reagent (Invitrogen, USA) according to the manufacturer's instructions. CDNA was generated by using $2 \mu \mathrm{g}$ total RNA, $0.5 \mu \mathrm{l}$ RNasin $(50 \mathrm{U} / \mu \mathrm{l}), 1 \mu \mathrm{l}$ random primers $(500 \mu \mathrm{g} / \mathrm{ml}), 2 \mu \mathrm{l}$ $10 \mathrm{mM}$ dNTP, $4 \mu \mathrm{l} 5 \mathrm{X}$ reverse transcription reaction buffer (Tris-HCl 250 mM, pH 8.3; KCl 375 mM; $\mathrm{MgCl}_{2} 15 \mathrm{mM}$ ) and $1 \mu \mathrm{l} \mathrm{M-MLV} \mathrm{reverse} \mathrm{transcriptase}(200 \mathrm{U} / \mu \mathrm{l})$ and were mixed to a final volume of $20 \mu \mathrm{l}$ with DEPC water (Kangwei Corporation, Beijing, China). First, the reaction mixture was incubated at $37^{\circ} \mathrm{C}$ for $55 \mathrm{~min}, 94^{\circ} \mathrm{C}$ for $5 \mathrm{~min}$ (to deactivate reverse transcriptase) and then frozen at $-20^{\circ} \mathrm{C}$.

Primer Express 5.0 was used to design the following primers: PTEN forward primer, 5'-GGA AAG GAC GGA CTG GTG TA-3' and reverse primer, 5'-TGC CAC TGG TCT GTA ATC CA-3' (101 bp amplicon); GAPDH forward primer, 5'-GGC AAG TTC AAC GGC ACA G-3' and reverse primer, 5'-CGC CAG TAG ACT CCA CGA CAT-3' (122 bp amplicon). The primers were synthesized by Saibaisheng Gene Co., Ltd. (Beijing, China). Real-time fluorescent quantitative PCR was performed using $12.5 \mu \mathrm{l} 2$ UltraSYBR Mixture, $0.5 \mu \mathrm{l}$ forward primer, $0.5 \mu \mathrm{l}$ reverse primer and $2 \mu \mathrm{l} \mathrm{cDNA}$ template in a total volume of $25 \mu \mathrm{l}$. Reaction conditions were: $95^{\circ} \mathrm{C}$ for $10 \mathrm{~min}, 1$ cycle; $95^{\circ} \mathrm{C}$ for $15 \mathrm{sec}, 60^{\circ} \mathrm{C}$ for $1 \mathrm{~min}, 35$ cycles. An ABI Prism 7700 real-time fluorescent quantitative PCR thermal cycler (Applied Biosystems, Foster City, CA, USA) was used. The mRNA expression of the PTEN gene was normalized to GAPDH. 
A Fresh livers

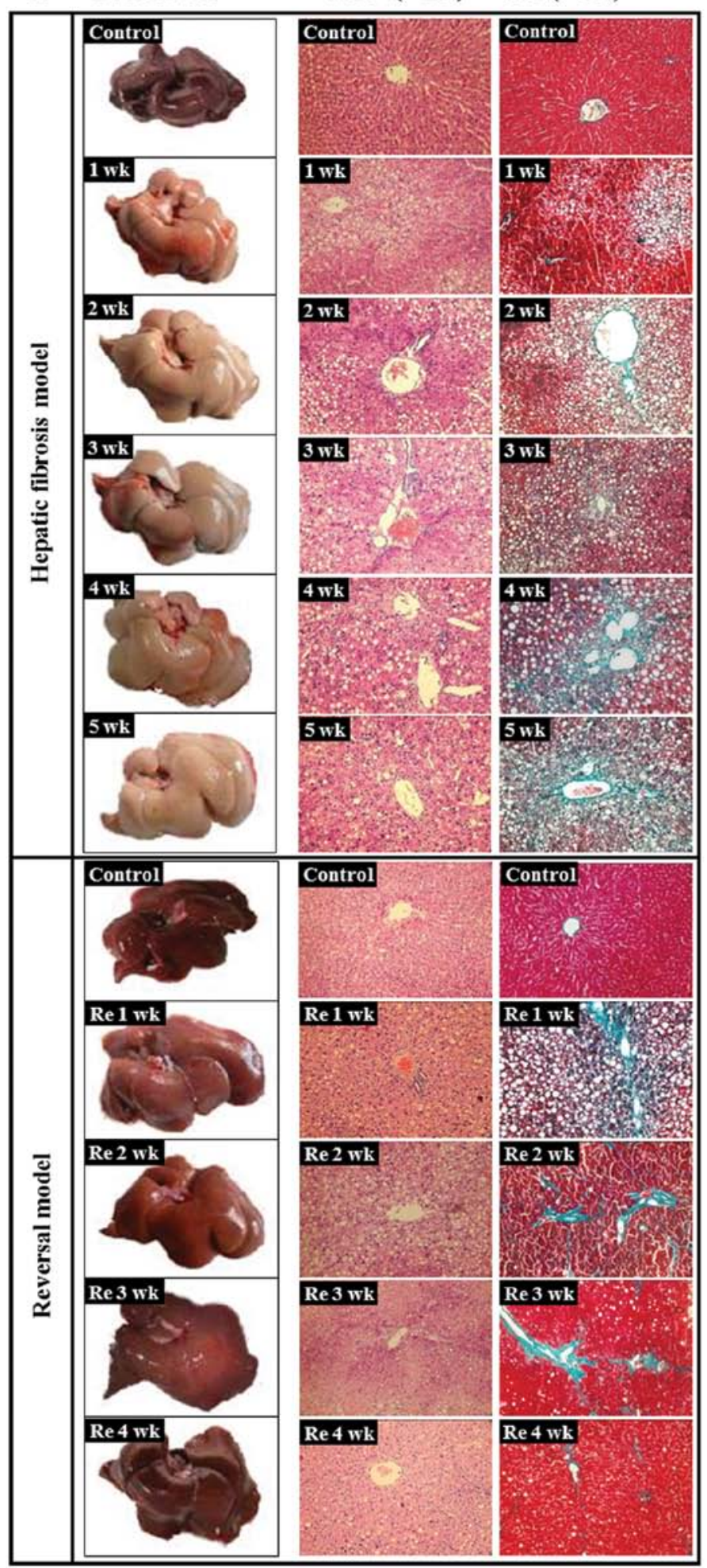

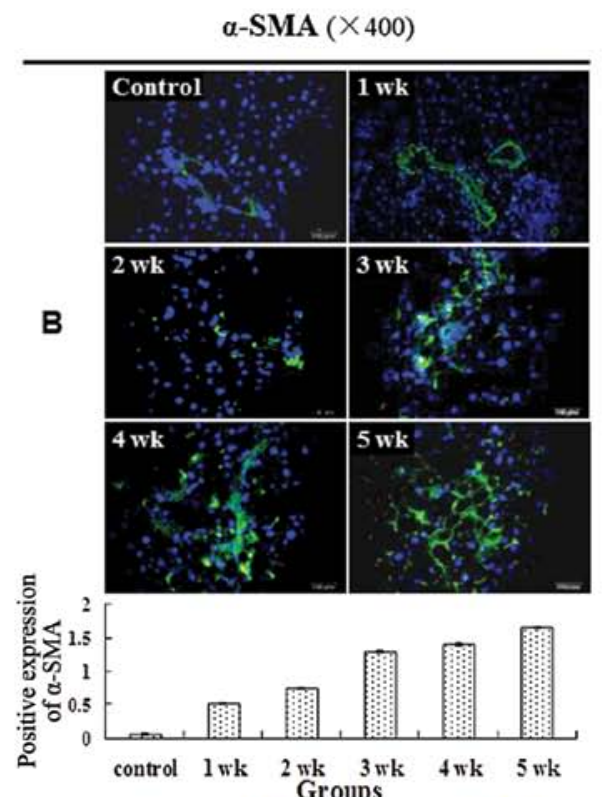
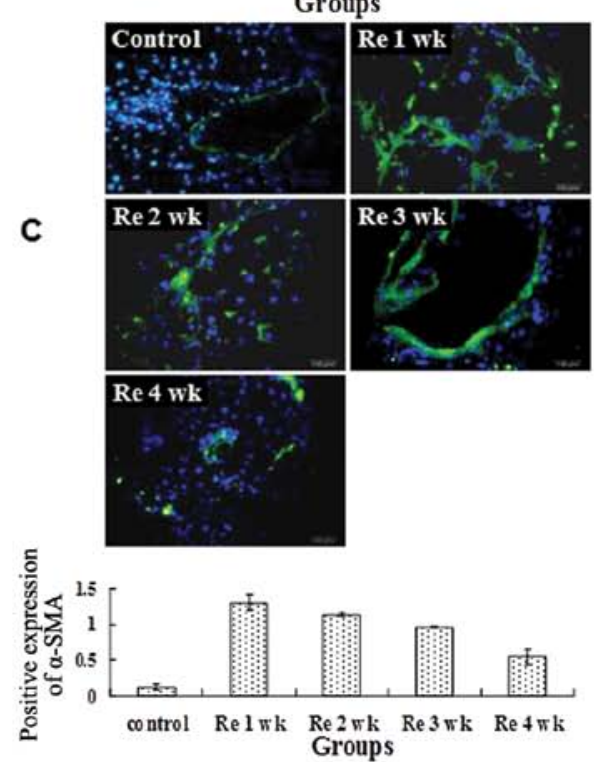

Figure 1. Hepatic fibrosis model and reversal model were successfully established. (A) Representative images of the fresh livers without fixation demonstrated that the livers of the control group were smooth, lustrous and reddish. However, after treatment with $\mathrm{CCl}_{4}$, the liver was turgescent and its surface appeared coarse and relatively bloodless. The above changes became more evident from 1 to 5 weeks. At the end of $\mathrm{CCl}_{4}$ treatment, the liver surfaces, although slightly coarse, became more reddish, lustrous and blood-filled and gradually returned to normal from Re 1 to 4 weeks. Liver sections stained with $\mathrm{H} \& \mathrm{E}$ demonstrated that hepatic cells were swollen and fatty degeneration, necrosis and regeneration were evident. Continuous $\mathrm{CCl}_{4}$ treatment led to worse liver damage from 1 to 5 weeks in the model group. After $\mathrm{CCl}_{4}$ treatment, the above histopathological changes gradually returned to normal. Liver sections stained with MT demonstrated that fibrosis spread from the vascular smooth muscle cells to the portal area and damaged hepatic cells; the latter appeared as fatty degeneration, necrosis and regeneration. The continuous $\mathrm{CCl}_{4}$ treatment led to an enlarged fibrotic area from 1 to 5 weeks in the model group. After termination of $\mathrm{CCl}_{4}$ treatment, the damaged hepatic cells gradually returned to normal and the fibrotic tissue area decreased from Re 1 to 4 weeks. (B) In the normal rat liver, $\alpha$-SMA was occasionally detected in vascular smooth muscle cells and the expression level was low, revealing limited activation of $\mathrm{HSCs}$. After $\mathrm{CCl}_{4}$ administration, the $\alpha$-SMA spread to the portal area, demonstrating an increased activation of HSCs. Immunofluorescence staining was used to discover a significant increased expression of $\alpha$-SMA from 1 to 5 weeks in the model group $(0.51 \pm 0.02,0.74 \pm 0.02,1.29 \pm 0.02,1.40 \pm 0.01,1.65 \pm 0.02)(\mathrm{P}<0.01)$, compared with the control group $(0.07 \pm 0.01)(\mathrm{P}<0.01)$. (C) A significant decrease in expression of $\alpha$-SMA was noted from Re 1 to 4 weeks $(\mathrm{P}<0.05)$. 
A
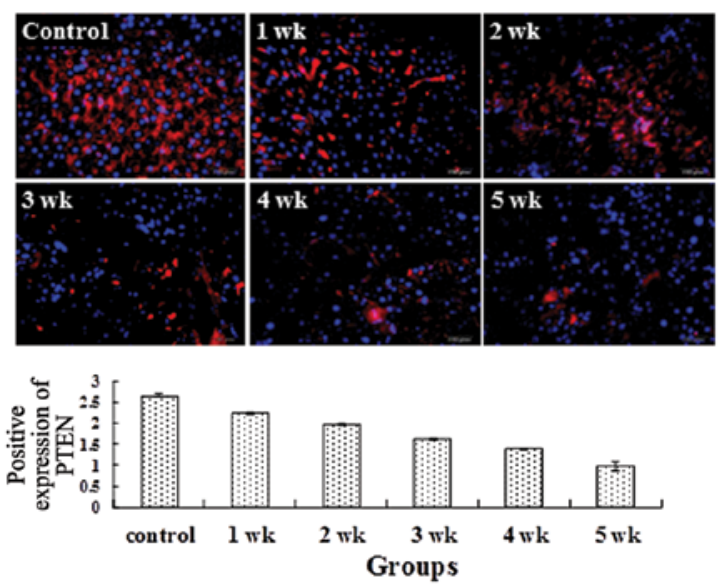

B
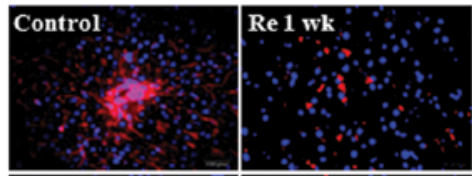

$\operatorname{Re} 2$ wk
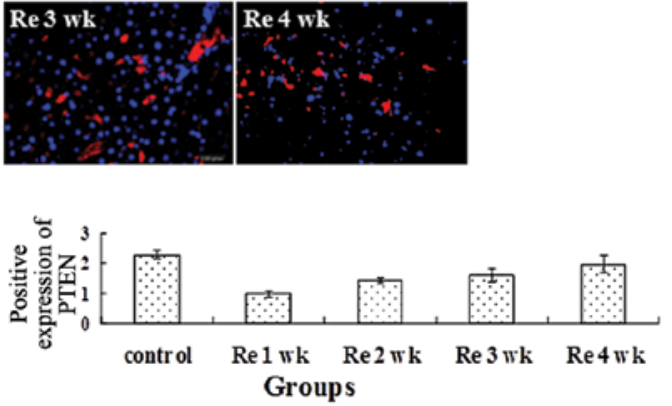

C
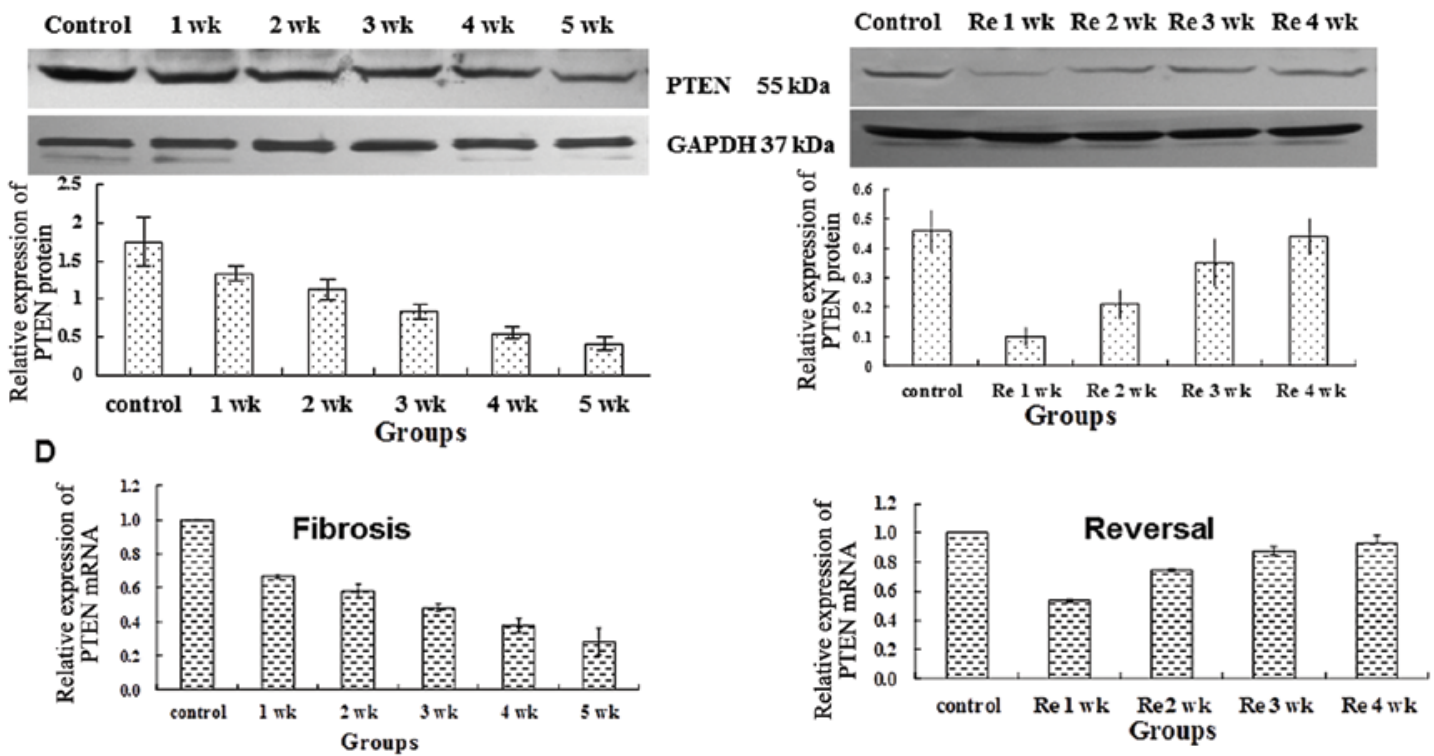

Figure 2. PTEN protein and mRNA decreased in the model groups and increased in the reversal groups. (A and B) In the normal rat liver tissue, PTEN protein as indicated in red fluorescence existed in widespread areas. Its expression decreased significantly after $\mathrm{CCl}_{4}$ administration in the model groups and increased after the end of $\mathrm{CCl}_{4}$ administration in the reversal groups. (C and D) The relative expression of PTEN protein and mRNA significantly decreased from 1 to 5 weeks $(\mathrm{P}<0.01)$ and increased from Re 1 to 4 weeks $(\mathrm{P}<0.01)$.

Statistical treatment. Data are presented as the means \pm SD and analyzed with SPSS 18.0 software. The statistical analyses that were performed included one-way ANOVA, the LSD test and Pearson's correlation analysis. $\mathrm{P}<0.05$ was considered to indicate a statistically significant difference.

\section{Results}

Gross and histopathological characterization of $\mathrm{CCl}_{4^{-}}$ induced liver fibrosis. $\mathrm{CCl}_{4}$-induced hepatic injury in rats has been used as a model system to study liver fibrogenesis. The gross appearance of the liver became progressively turgescent, coarse and relatively bloodless over 5 weeks of $\mathrm{CCl}_{4}$ administration (Fig. 1A). In contrast, withdrawal of $\mathrm{CCl}_{4}$ improved gross liver morphology (reduced surface coarseness and increased reddish color) over time (Fig. 1A).

Histologic examination of the $\mathrm{CCl}_{4}$-treated liver revealed swelling of the hepatocytes, fatty degeneration, necrosis and regeneration, particularly with continued $\mathrm{CCl}_{4}$ administration (Fig. 1A). After termination of $\mathrm{CCl}_{4}$ treatment, the above histopathological changes returned to normal over time (Fig. 1A).

To determine whether there was increased collagen deposition, MT staining was performed. Increased MT staining in the periportal region was discovered with $\mathrm{CCl}_{4}$ administration from 1 to 5 weeks (Fig. 1A). At the end of $\mathrm{CCl}_{4}$ administration, the damaged hepatic cells gradually returned to normal and the degree of MT staining was reverted to the baseline level at Re 4 weeks (Fig. 1A).

Activation profile of HSCs with liver fibrosis. To assess the role of HSCs, the expression analysis of $\alpha$-SMA was performed. On activation, HSCs undergo proliferation and differentiation, becoming myofibroblast-like $\alpha$-smooth muscle actin ( $\alpha$-SMA)positive cells that produce ECM proteins, particularly type I collagen (2). In the normal rat liver, $\alpha$-SMA was occasionally detected in vascular smooth muscle cells and the expression 


\section{A}

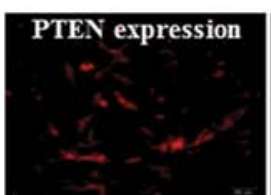

B
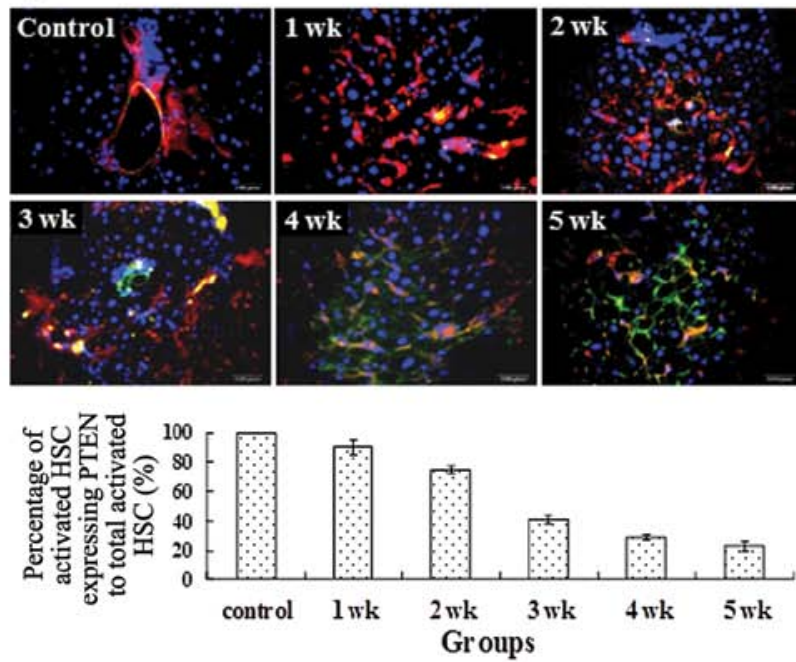
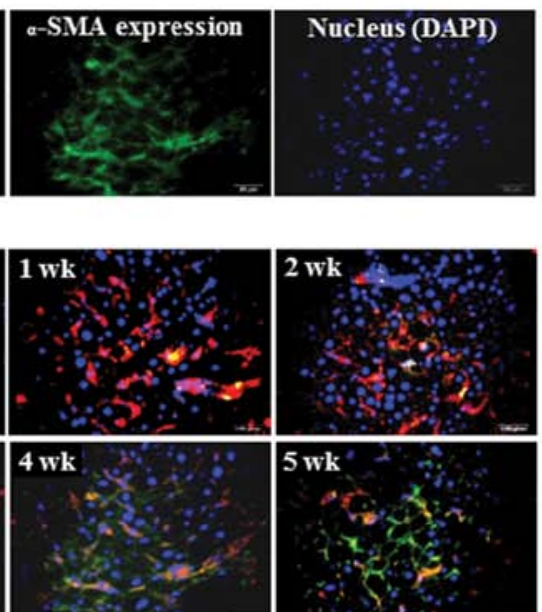

5 wk

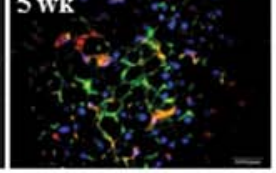

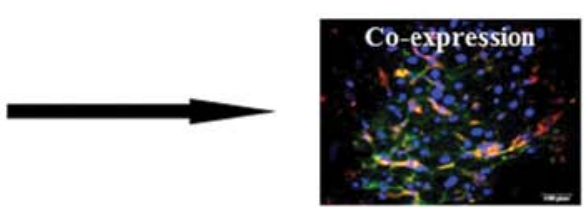

C
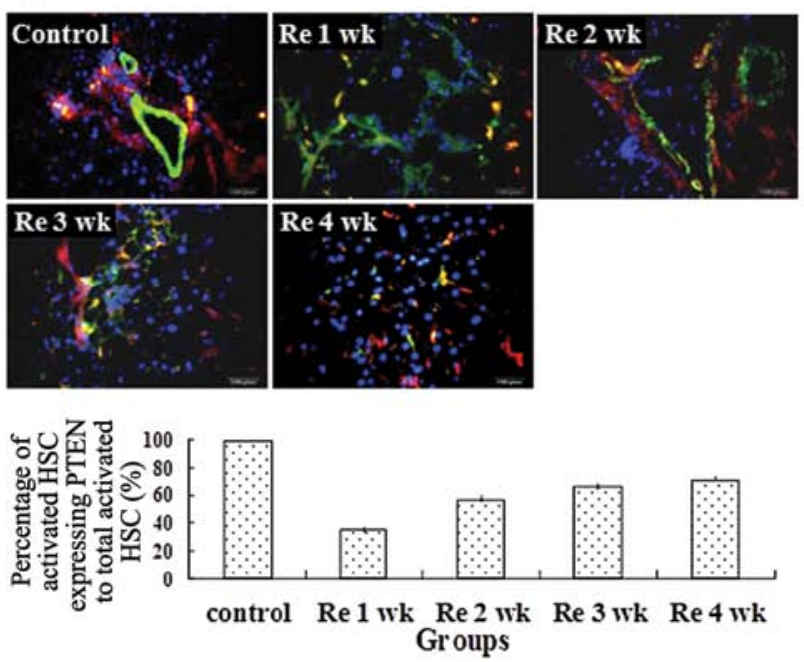

Figure 3. PTEN expression in activated HSCs decreased in the model groups and increased in the reversal groups. (A) PTEN protein is indicated by red fluorescence, $\alpha$-SMA by green fluorescence and the colocalization of PTEN and $\alpha$-SMA in activated HSCs in rat fibrogenic hepatic tissues are indicated in the yellow area. (B and C) From 1 to 5 weeks, the percentage of activated HSCs expressing PTEN to total activated HSCs decreased in the model group and an increasing percentage was noted from $\operatorname{Re} 1$ to 4 weeks $(\mathrm{P}<0.01)$.

A
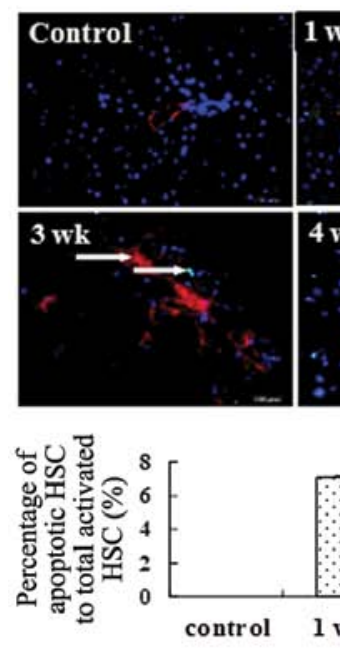
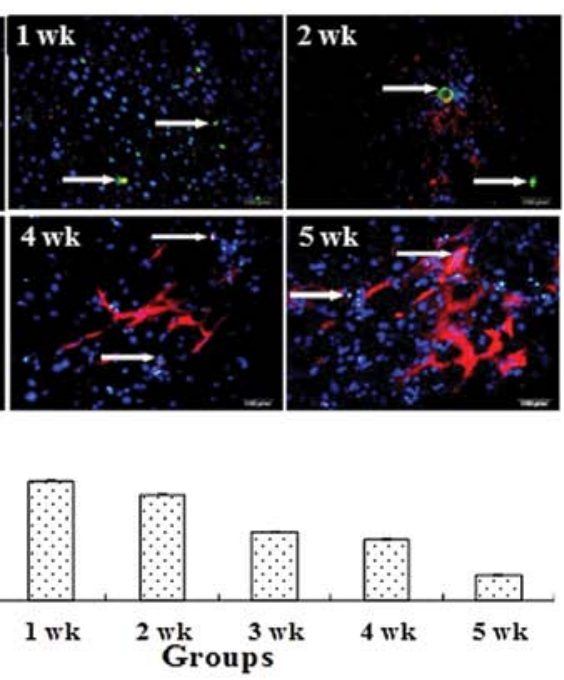

B
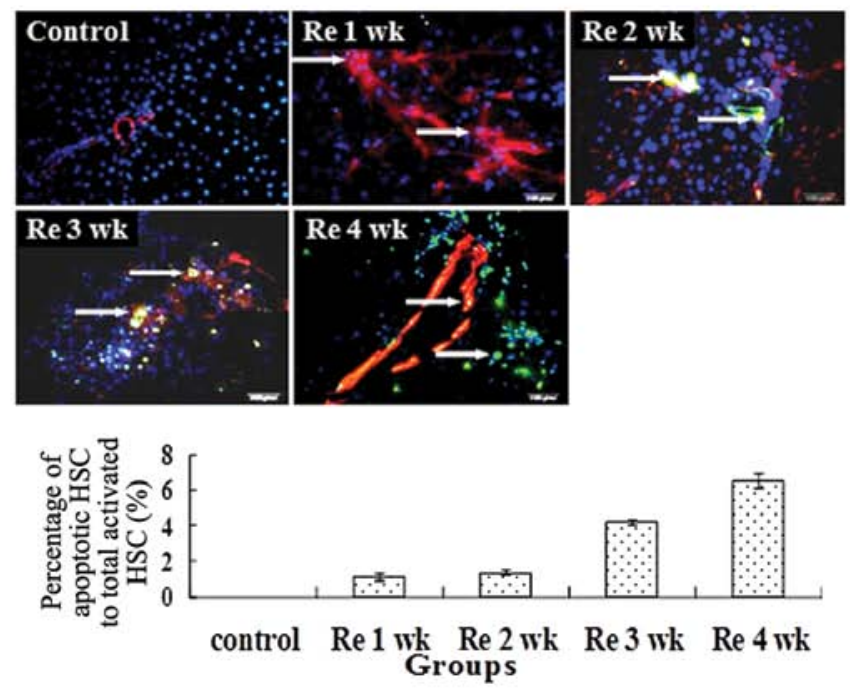

Figure 4. The apoptotic index of activated HSCs decreased in the model groups and increased in the reversal groups. Activated HSCs expressing $\alpha$-SMA are indicated by red fluorescence and apoptotic cells by green fluorescence using TUNEL staining. The two images were then combined to display the apoptotic activated HSCs indicated in yellow. The percentage of total activated HSCs (apoptotic index) significantly decreased from 1 to 5 weeks in the model group and increased from Re 1 to 4 weeks $(\mathrm{P}<0.01)$.

level was low, revealing a limited activation of HSCs (Fig. 1B). After $\mathrm{CCl}_{4}$ administration, the $\alpha$-SMA spread to the portal area, demonstrating an increased activation of HSCs. Compared with the control group $(0.07 \pm 0.01)$, treatment with $\mathrm{CCl}_{4}$ led to a significant and progressively increased expression of $\alpha$-SMA from 1 to 5 weeks $(0.51 \pm 0.02,0.74 \pm 0.02,1.29 \pm 0.02,1.40 \pm 0.01$, $1.65 \pm 0.02$; $\mathrm{P}<0.01$ ) (Fig. $1 \mathrm{~B}) . \mathrm{CCl}_{4}$ withdrawal resulted in a significantly decreased $\alpha$-SMA expression between Re 1,2 , 3 and 4 weeks $(1.31 \pm 0.11,1.14 \pm 0.03,0.97 \pm 0.01,0.55 \pm 0.11$; $\mathrm{P}<0.05$ ) (Fig. 1C).

PTEN expression declines during liver fibrogenesis and increases during reversal of fibrosis. In normal rat liver tissue recent reports demonstrate that there is an inverse correlation 
between PTEN and fibrogenesis in pulmonary fibrosis (7). We then assessed the potential association between PTEN and liver fibrosis. In the normal rat liver tissue, PTEN protein existed in widespread areas (Fig. 2A). As the normal hepatic cells were damaged by the administration of $\mathrm{CCl}_{4}$, the PTEN expression decreased significantly from 1 to 5 weeks $(2.23 \pm 0.02,1.96 \pm 0.03,1.61 \pm 0.02,1.37 \pm 0.02,0.98 \pm 0.01$; $\mathrm{P}<0.01)$, compared with the control group $(2.67 \pm 0.02)$ (Fig. 2A). Withdrawal of $\mathrm{CCl}_{4}$ led to the progressive increase in PTEN protein expression from Re 1 to 4 weeks $(1.01 \pm 0.11$, $1.46 \pm 0.09,1.62 \pm 0.22,1.99 \pm 0.30 ; \mathrm{P}<0.01)$; however it remained less the normal level of the control group (2.31 \pm 0.12$)$ (Fig. 2B).

We next used western blot analysis and real-time (RT)-PCR to quantitate the expression change of PTEN. Compared to the control, the relative expression levels of PTEN/GAPDH in rat hepatic tissues were significantly lower at 1,2,3,4 and 5 weeks. As $\mathrm{CCl}_{4}$ was withdrawn, PTEN expression increased over time (Fig. 2C). However, PTEN expression remained significantly lower compared to the control. Consistent with the changes in PTEN expression at the protein level, the relative expression levels of PTEN mRNA in rat liver tissues were significant lower at 1, 2, 3, 4 and 5 weeks in the model group compared to the control group (Fig. 2C). Withdrawing $\mathrm{CCl}_{4}$ led to a gradual increase in PTEN expression over time (Fig. 2C). Collectively, we demonstrate that PTEN expression declines during liver fibrogenesis and increases during reversal of fibrosis.

PTEN expression in activated HSCs. The potential role of PTEN in HSCs during liver fibrogenesis and reversal was investigated. We used $\alpha$-SMA as a marker of HSC activation and measured the co-localization with PTEN. Administration of $\mathrm{CCl}_{4}$ led to reduced immunofluorescent staining of PTEN in activated HSCs from 1 to 5 weeks (Fig. 3A). During reversal of fibrosis, the percentage of activated HSCs expressing PTEN increased (Fig. 3B and C).

Since the number of activated HSCs was dynamic during fibrogenesis and its reversal, we assessed whether apoptosis was one of the mechanisms required to regulate the number of activated HSCs. TUNEL staining demonstrated that the percentage of apoptotic activated HSCs to total activated HSCs decreased from 1 to 5 weeks with the administration of $\mathrm{CCl}_{4}$ (Fig. 4A). By contrast, the reduced number of activated HSCs was correlated with the increased apoptotic activity during withdrawal of $\mathrm{CCl}_{4}$ (Fig. 4B).

Correlation analysis. Pearson's correlation analysis discovered a significant negative correlation between PTEN and $\alpha$-SMA expression $(r=-0.979, \mathrm{P}<0.05)$. Significant positive correlations were observed between PTEN expression to PTEN-positive activated HSCs $(r=0.962, \mathrm{P}<0.05)$ and PTEN expression and the apoptotic index of activated HSCs $(r=0.991, \mathrm{P}<0.05)$. Collectively, our data demonstrate that PTEN is negatively correlated with the activation of HSCs and positively correlated with the apoptosis of activated HSCs.

\section{Discussion}

In addition to its role in cell proliferation and tumor biology (11-14), recent studies have unveiled a novel role of PTEN in fibrosis including diffuse systemic sclerosis (15) and idiopathic pulmonary interstitial fibrosis (16). Additionally, recent studies have shown that PTEN had a negative relation with the activation and proliferation of HSCs in fibrotic hepatic tissues induced by bile duct ligation (BDL) (8). The upregulation of PTEN was found to inhibit the activation and proliferation in HSCs of fresh isolated rats $(17,18)$ and induce apoptosis of activated HSCs in vitro (9). We used the $\mathrm{CCl}_{4}$ hepatic fibrosis model to further investigate the potential role of PTEN in fibrogenesis.

Using immunofluorescent staining, PTEN protein is present mostly in cytolymph with occasional expression in the nucleus. The expression of PTEN is dynamic during fibrogenesis and reversal of fibrosis. Consistent with previous results $(8,9)$, we revealed that PTEN expression was reduced in activated HSCs during fibrogenesis and increased in activated HSCs during reversal of fibrosis.

HSCs are typically found in the space of Disse in a quiescent state (19). HSCs may be activated to myofibroblasts expressing $\alpha$-SMA by several stimuli such as cytokines and inflammatory mediators $(20,21)$. Activated HSCs migrate to and proliferate in sites of liver injury $(20,21)$, synthesize ECM components and upregulate the expression levels of $\alpha$-SMA and collagen matrices (22). Hence, $\alpha$-SMA is a marker of HSC activation and proliferation. We demonstrated that in normal rat liver, $\alpha$-SMA was occasionally detected in vascular smooth muscle cells and the expression level was low, revealing limited activation of $\mathrm{HSCs}$. After $\mathrm{CCl}_{4}$ administration, the $\alpha$-SMA spread to the portal area, demonstrating an increased activation of HSCs. During reversal of fibrosis, the number of activated HSCs declined via apoptosis. Together, our results further implicate that PTEN may participate in the pathogenesis of hepatic fibrosis by affecting the activation state and apoptosis of HSCs. We propose that PTEN may inhibit the activation and proliferation of HSCs whereas its downregulation promotes the activation and proliferation of HSCs, leading to fibrogenesis

It has been reported that PTEN may regulate the activity of fibroblasts (23); low expression of PTEN may promote the differentiation of myofibroblasts and enhance the morbidity of pulmonary interstitial fibrosis (7). High expression of PTEN may inhibit the proliferation and migration of hepatocarcinoma cells (24) and induce apoptosis (25). Our previous study also revealed that the upregulation of PTEN expression induced apoptosis of activated HSCs in vitro (9). In this study, we demonstrated that during reversal of fibrosis, elimination of activated HSCs by apoptosis occurred. It has been previously shown that aspergillin, sulfasalazine and anti-TIMP1 antibodies promoted the reversal of liver fibrosis by inducing the apoptosis of activated HSCs (26). Since PTEN expression is positively correlated with the apoptosis of activated HSCs, one of the potential molecular mechanism for reversal of fibrosis is possibly through apoptotic elimination of activated HSCs through PTEN expression.

In conclusion, our data demonstrated that the dynamic expression of PTEN in rat liver tissues had a significant negative correlation with the activation and proliferation of HSCs and had a significant positive correlation with the apoptosis of activated HSCs in vivo. The association of PTEN expression with HSC activation and apoptosis implicates its 
role in liver fibrosis and its reversal. Accumulating evidence from our current study and previously published data suggest that PTEN is a novel and relevant target for the treatment of liver fibrosis.

\section{Acknowledgements}

This study was supported by the National Natural Science Foundation of China (no. 30872513), the Hebei Provincial Natural Science Foundation of China (C2010000565) and the Department of Science and Technology in Hebei Province (09966108D). The authors would like to thank the Foundations for their support.

\section{References}

1. Povero D, Busletta C, Novo E, di Bonzo LV, Cannito S Paternostro $\mathrm{C}$ and Parola M: Liver fibrosis: a dynamic and potentially reversible process. Histol Histopathol 25: 1075-1091, 2010.

2. Friedman SL: Mechanisms of hepatic fibrogenesis. Gastroenterology 134: 1655-1669, 2008.

3. Friedman SL: Hepatic stellate cells: protean, multifunctional, and enigmatic cells of the liver. Physiol Rev 88: 125-172, 2008

4. Lakner AM, Walling TL, McKillop IH and Schrum LW: Altered aquaporin expression and role in apoptosis during hepatic stellate cell activation. Liver Int 31: 42-51, 2011.

5. Kisseleva T and Brenner DA: Hepatic stellate cells and the reversal of fibrosis. J Gastroenterol Hepatol 21 (Suppl 3): S84-S87, 2006.

6. Li J, Yen C, Liaw D, et al: PTEN, a putative protein tyrosine phosphatase gene mutated in human brain, breast, and prostate cancer. Science 275: 1943-1947, 1997.

7. White ES, Atrasz RG, Hu B, et al: Negative regulation of myofibroblast differentiation by PTEN (phosphatase and tensin homolog deleted on chromosome 10). Am J Respir Crit Care Med 173: 112-121, 2006.

8. Hao LS, Zhang XL, An JY, et al: PTEN expression is downregulated in liver tissues of rats with hepatic fibrosis induced by biliary stenosis. APMIS 117: 681-691, 2009.

9. Hao LS, Zhang XL, An JY, et al: Adenoviral transduction of PTEN induces apoptosis of cultured hepatic stellate cells. Chin Med J (Engl) 122: 2907-2911, 2009.

10. Montfort I and Perez-Tamayo R: Collagenase in experimental carbon tetrachloride cirrhosis of the liver. Am J Pathol 92. 411-420, 1978

11. Cotler SJ, Hay N, Xie H, Chen ML, Xu PZ, Layden TJ and Guzman G: Immunohistochemical expression of components of the Akt-mTORC1 pathway is associated with hepatocellular carcinoma in patients with chronic liver disease. Dig Dis Sci 53 844-849, 2008
12. Lai JP, Bao S, Davis IC and Knoell DL: Inhibition of the phosphatase PTEN protects mice against oleic acid-induced acute lung injury. Br J Pharmacol 156: 189-200, 2009.

13. Peyrou M, Bourgoin L and Foti M: PTEN in non-alcoholic fatty liver disease/non-alcoholic steatohepatitis and cancer. Dig Dis 28: 236-246, 2010

14. Dasari VR, Kaur K, Velpula KK, et al: Upregulation of PTEN in glioma cells by cord blood mesenchymal stem cells inhibits migration via downregulation of the PI3K/Akt pathway. PLoS One 5: e10350, 2010

15. Bu S, Asano Y, Bujor A, Highland K, Hant F and Trojanowska M: Dihydrosphingosine 1-phosphate has a potent antifibrotic effect in scleroderma fibroblasts via normalization of phosphatase and tensin homolog levels. Arthritis Rheum 62: 2117-2126, 2010.

16. Xia H, Khalil W, Kahm J, Jessurun J, Kleidon J and Henke CA Pathologic caveolin-1 regulation of PTEN in idiopathic pulmonary fibrosis. Am J Pathol 176: 2626-2637, 2010.

17. Sato W, Horie Y, Kataoka E, et al: Hepatic gene expression in hepatocyte-specific Pten deficient mice showing steatohepatitis without ethanol challenge. Hepatol Res 34: 256-265, 2006.

18. Takashima M, Parsons CJ, Ikejima K, Watanabe S, White ES and Rippe RA: The tumor suppressor protein PTEN inhibits rat hepatic stellate cell activation. J Gastroenterol 44: 847-855, 2009.

19. Geerts A: History, heterogeneity, developmental biology, and functions of quiescent hepatic stellate cells. Semin Liver Dis 21: 311-335, 2001.

20. Bataller R and Brenner DA: Hepatic stellate cells as a target for the treatment of liver fibrosis. Semin Liver Dis 21: 437-451, 2001.

21. Friedman SL: Molecular regulation of hepatic fibrosis, an integrated cellular response to tissue injury. J Biol Chem 275: 2247-2250, 2000.

22. Arthur MJ: Fibrogenesis II. Metalloproteinases and their inhibitors in liver fibrosis. Am J Physiol Gastrointest Liver Physiol 279: G245-G249, 2000.

23. Nho RS, Xia H, Diebold D, Kahm J, Kleidon J, White E and Henke CA: PTEN regulates fibroblast elimination during collagen matrix contraction. J Biol Chem 281: 33291-33301, 2006.

24. Tian T, Nan KJ, Guo H, et al: PTEN inhibits the migration and invasion of HepG2 cells by coordinately decreasing MMP expression via the PI3K/Akt pathway. Oncol Rep 23: 1593-1600, 2010.

25. Cao LQ, Chen XL, Wang Q, et al: Upregulation of PTEN involved in rosiglitazone-induced apoptosis in human hepatocellular carcinoma cells. Acta Pharmacol Sin 28: 879-887, 2007.

26. Brenner DA: Molecular pathogenesis of liver fibrosis. Trans Am Clin Climatol Assoc 120: 361-368, 2009. 